\title{
Impact of caregiver and parenting status on time trade-off and standard gamble utility scores for health state descriptions
}

\author{
Louis S Matza ${ }^{1 *}$, Kristina S Boye ${ }^{2}$, David H Feeny ${ }^{3}$, Joseph A Johnston ${ }^{4}$, Lee Bowman ${ }^{5}$ and Jessica B Jordan ${ }^{6}$
}

\begin{abstract}
Background: The purpose of this study was to examine the effect of caregiver status on time trade-off (TTO) and standard gamble (SG) health state utility scores. Respondents were categorized as caregivers if they reported that either children or adults depended on them for care.

Methods: This study was a secondary analysis of data from three studies in which general population samples rated health state descriptions. Study 1: UK; four osteoarthritis health states. Study 2: UK; three adult ADHD health states. Study 3: US; 16 schizophrenia health states. All three studies included time trade-off assessment. Study 1 also included standard gamble. Descriptive statistics were calculated to examine willingness to trade in $\Pi \mathrm{O}$ or gamble in SG. Utilities for caregivers and non-caregivers were compared using t-tests and ANCOVA models.

Results: There were 364 respondents including 106 caregivers ( $n=30,47$, and 29 in Studies 1, 2, and 3) and 258 non-caregivers. Most caregivers were parents of dependent children (78.3\%). Compared to non-caregivers, caregivers had more responses at the ceiling (i.e., utility $=0.95$ ), indicating less willingness to trade time or gamble. All utilities were higher for caregivers than non-caregivers (mean utility difference between groups: 0.07 to 0.16 in Study 1 TTO; 0.03 to 0.17 in Study 1 SG; 0.06 to 0.10 in Study 2 TTO; 0.11 to 0.22 in Study 3 TTO). These differences were statistically significant for at least two health states in each study $(p<0.05)$. Results of sensitivity analyses with two caregiver subgroups (parents of dependent children and parents of any child regardless of whether the child was still dependent) followed the same pattern as results of the primary analysis. The parent subgroups were generally less willing to trade time or gamble (i.e., resulting in higher utility scores) than comparison groups of non-parents.
\end{abstract}

Conclusions: Results indicate that caregiver status, including being a parent, influences responses in time trade-off health state valuation. Caregivers (i.e., predominantly parents) were less willing than non-caregivers to trade time, resulting in higher utility scores. This pattern was consistent across multiple health states in three studies. Standard gamble results followed similar patterns, but with less consistent differences between groups. It may be useful to consider parenting/caregiving status when collecting, interpreting, or using utility data because this demographic variable could influence results.

Keywords: Utility, Time trade-off, Standard gamble, Caregiver, Parenting, Cost-utility

\footnotetext{
* Correspondence: louis.matza@evidera.com

'Senior Research Scientist, Outcomes Research, Evidera, Bethesda, MD, USA

Full list of author information is available at the end of the article
} 


\section{Background}

A cost-utility model is a type of cost-effectiveness analysis that incorporates the preferences of individuals for various health states, and these preferences are quantified in terms of utilities [1-3]. Healthcare policy and reimbursement decisions are often directly informed by results of cost-utility models, and the results of these models depend on the utility scores. Therefore, it is important to understand factors that may influence respondents' decisions in tasks designed to elicit utilities.

There is a substantial body of research on respondent characteristics that may influence health state preferences and utility scores. Characteristics that have been found in at least one study to influence utilities include experience of the health state being rated $[4,5]$, education level $[6,7]$, religious beliefs [8-10], age [7,11,12], gender [11], marital status [11], race [13], and culture [14].

One participant characteristic that has received relatively little attention is whether the respondent is the caregiver of other individuals such as young children. One previous study examined the extent to which being a mother of a young child influenced responses to time trade-off (TTO) utility assessment [15]. A group of 30 mothers was compared to a large general population sample, and results indicated that the mothers were not willing to trade as much time as the general population sample. Thus, the small sample of mothers had significantly higher mean utilities for four generic EQ-5D health states than the general population. The authors interpreted this difference as suggesting that parenting could affect one's extrinsic goals, which could influence preferences during health state valuation.

The purpose of the current study was to analyze data from three utility studies to examine further the influence of caregiver status, including being a parent, on utilities. Because these three utility studies were conducted with different health states, the current analysis can provide insight into whether the impact of caregiver status is consistent across multiple samples and health states representing a range of health conditions. It was expected that caregivers, compared to non-caregivers, would be more resistant to trading time in TTO utility assessment and more resistant to gambling in standard gamble utility assessment. These tendencies were expected to result in higher utilities for the same health states for caregivers than for non-caregivers.

\section{Methods}

\section{Overview of study design}

The current study is a secondary analysis of data from three studies in which general population respondents completed utility interviews to rate a series of health state descriptions. In Study 1, participants in London rated four osteoarthritis health states in time trade-off and standard gamble (SG) tasks. These health states varied by severity of osteoarthritis symptoms. In Study 2, participants in London and Edinburgh rated three adult attention-deficit/hyperactivity disorder (ADHD) health states in TTO interviews. These health states described treatment response, treatment non-response, and untreated adult ADHD. In Study 3, participants in San Francisco rated 16 health states in TTO interviews. These health states described schizophrenia and antipsychotic treatment with a range of adverse events. All TTO utility assessment procedures were consistent across the three studies, including the use of a 10 -year time horizon. Table 1 provides an overview of the three studies used in this secondary analysis.

For the current analyses, participants were categorized as caregivers or non-caregivers. Respondents were asked "Do you have any children?" If they responded "yes," they were asked "How many of these children still depend on you to care for them?" Finally, they were asked "Are there any other people besides children who depend on you to care for them (for example, elderly or disabled relatives)?" Participants were considered to be caregivers if they reported that at least one child or one adult depended on them for care. Otherwise, they were classified as non-caregivers.

\section{Participants}

In all three studies, participants were required to be (1) at least 18 years old; (2) able to understand the assessment procedures; and (3) able and willing to give written informed consent. Participants were not eligible if they had cognitive impairment, hearing difficulty, visual impairment, severe psychopathology, or insufficient knowledge of English that could interfere with the ability to complete study measures. Inclusion criteria did not specify any particular clinical characteristics, which is consistent with recommendations from agencies like the UK's National Institute for Health and Care Excellence (NICE), most of which prefer that utilities represent general population preferences [16-18]. Participants were recruited through newspaper and online classified advertisements.

\section{Study 1}

A total of 197 potential participants responded to advertisements, and 109 of these were reached for screening. Of the 109 screened participants, two were ineligible, 101 were scheduled for interviews, and 81 attended interviews in London, UK. Of the 81 participants, one was unable to complete the utility interview procedures. Therefore, the Study 1 analysis was conducted with the 80 participants who completed the interview (i.e., $40.6 \%$ of individuals who initially responded to the advertisements).

\section{Study 2}

A total of 396 potential participants responded to advertisements, and 203 of these were reached for screening. 
Table 1 Data sources for the current secondary analysis

\begin{tabular}{lccc}
\hline Study characteristics & Study $\mathbf{1}$ & Study $\mathbf{2}$ & Study $\mathbf{3}$ \\
\hline $\begin{array}{l}\text { Number of health states } \\
\text { Description of health states }\end{array}$ & 4 & 3 & 16 \\
Utility assessment method & $\begin{array}{c}\text { Osteoarthritis: three severity levels, } \\
\text { plus one adverse event }\end{array}$ & $\begin{array}{c}\text { Adult ADHD: responder, } \\
\text { non-responder, untreated }\end{array}$ & $\begin{array}{c}\text { Schizophrenia with a range of } \\
\text { treatment-related adverse events }\end{array}$ \\
$\begin{array}{l}\text { Geographic location } \\
\text { Dates of data collection }\end{array}$ & London (UK) & TTO & TTO \\
Sample size & November 2011 & London and Edinburgh (UK) & San Francisco (US) \\
Total & 80 & October 2012 & April-May 2012 \\
Caregivers & 30 & 158 & 126 \\
Non-caregivers & 50 & 47 & 29 \\
\hline
\end{tabular}

TTO = Time trade-off; SG = Standard gamble; UK = United Kingdom; US = United States; ADHD = Attention deficit/hyperactivity disorder.

Of the 203 screened participants, 199 were eligible, 174 were scheduled (88 in Edinburgh and 86 in London) for interviews, and 160 participants attended interviews (81 in Edinburgh and 79 in London). Two of the 160 participants were unable to complete the utility interview. Thus, a total of 158 completed utility interviews were used in the analyses (i.e., 39.9\% of individuals who initially responded to the advertisements), including 80 in Edinburgh and 78 in London.

\section{Study 3}

A total of 376 participants responded to the advertisements, and 202 of these were reached for screening. Of the 202 screened participants, 192 were eligible, 136 were scheduled for interviews, and 128 participants attended interviews in San Francisco, US. Two of the 128 participants were unable to complete the utility interview. Therefore, the Study 3 analysis was based on the 126 participants who completed the interview (i.e., $33.5 \%$ of individuals who initially responded to the advertisements).

\section{Health states \\ Study 1}

Four osteoarthritis health states associated with elective total hip arthroplasty were presented during the utility interview in Study 1. The first three health states described patients with mild (health state A), moderate (B), or severe (C) osteoarthritis of the hip. These health states were based on health states presented in two previous studies $[19,20]$, with minor edits made so that they would be appropriate for administration in the UK. A fourth health state (D) was identical to health state B, except for the addition of an adverse event: "Because of your medication, you occasionally have an upset stomach that causes discomfort and loss of appetite."

\section{Study 2}

Three adult ADHD health states were presented during the utility interview in Study 2: (A) Adult ADHD, receiving medication treatment, responder; (B): Adult $\mathrm{ADHD}$, receiving medication treatment, non-responder; and (C): Adult ADHD, untreated. These health states were drafted based on literature review, four clinician interviews (all four with MD degrees; two of whom also had $\mathrm{PhD}$ degrees), and pooled data from six clinical trials. The literature review was conducted first to ensure that the health states would be grounded in clinical evidence. This literature was then used to draft structured interview guides for the clinician interviews. The data from the clinical trials was examined to ensure that health states accurately described differences between treatment responders and non-responders.

\section{Study 3}

In order to estimate the disutility associated with adverse events commonly associated with antipsychotic treatment for schizophrenia, participants in Study 3 first rated health state A (i.e., the "basic health state"), which described stable schizophrenia without an adverse event. Then, 15 additional health states were rated, each including the basic health state plus one treatment-related adverse event. These health states were drafted based on literature review and a series of interviews with three clinicians (two with MD degrees and one with a PharmD) who had experience treating patients with schizophrenia.

\section{Utility interview procedures and scoring}

Interviews were conducted in London for Study 1, in London and Edinburgh for Study 2, and in San Francisco for Study 3 (see Table 1 for dates of data collection). All procedures were approved by an independent Institutional Review Board, and participants provided written informed consent prior to completing any part of each study.

First, participants in each study rated the health states in a visual analogue scale (VAS) or ranking exercise that was intended to introduce participants to each of the health state descriptions. In the VAS and ranking exercises, health states were presented to respondents in 
random order. Then, health state utilities were obtained using TTO methods for all three studies, in addition to SG methods for Study 1. In Study 1, half of the participants were randomly assigned to complete the TTO task first, while the other half completed the SG task first. In TTO and SG procedures, health states were presented in the order in which they were previously ranked by participants, proceeding from best to worst.

TTO and SG interviews followed generally accepted procedures, which have previously been described in detail $[1,21]$. In the TTO task, participants were offered a choice between spending 10 years in the health state being rated versus spending varying shorter amounts of time in full health, followed by death. Time was varied in 1-year increments. The utility score was calculated based on the choice in which the respondent was indifferent between $y$ years in the health state being evaluated (i.e., 10 years) and $x$ years in full health (followed by dead). The resulting utility estimate $(u)$ is calculated as $u=x / y$.

In the SG task in Study 1, participants were offered a choice between two alternatives, one that was certain and one that was uncertain. Choice $A$ was to remain in the health state being rated for 10 years. Choice B was the uncertain choice with two possible outcomes; either to (1) remain in full health for the 10-year period with a probability of $p$ or (2) death with a probability of $1-p$. Probability $p$ was varied in $10 \%$ increments until the participant was indifferent between choices $\mathrm{A}$ and $\mathrm{B}$, and the resulting utility score was calculated based on $p$ at this point of indifference.

In Study 1, precise utility scores were not obtained for health states considered worse than being dead for each respondent (i.e., negative utility scores). Therefore, "worse than dead" ratings in Study 1 are used in categorical analyses (e.g., counted among the frequencies of utilities less than 0.95), but not in continuous analyses which require precise utility scores.

In Studies 2 and 3, if participants indicated that a health state was worse than being dead, the interviewer altered the task so that respondents were offered a choice between immediate death (alternative 1) and a 10-year life span (alternative 2) beginning with varying amounts of time in the health state being rated, followed by full health for the remainder of the 10-year timeframe. For these health states that received negative utility scores, the current study used a bounded scoring approach, which is commonly used [21]. This scoring approach limits the score range of negative scores between 0 and -1 . To compute these utilities, analyses of Studies 2 and 3 used the Dolan [22] method as described by Rowen \& Brazier [1]. This method uses the formula $u=-x / t$, where $x$ is the number of years in full health, and $t$ is the total life span of alternative 2 in the TTO choice. In the current study, $t$ was 10 years, which is equal to the number of years in the health state being rated plus subsequent years in full health.

\section{Statistical analysis procedures}

Statistical analyses were completed using SAS version 8.12 (SAS Institute, Cary, NC). For each of the three studies, demographic variables, willingness to trade (in TTO), willingness to gamble (in SG for Study 1 only), and mean utilities are reported for two subgroups (caregivers and noncaregivers, categorized as described in the section above titled "Overview of Study Design"). Caregivers and noncaregivers were compared in terms of willingness to trade in TTO and willingness to gamble in SG with chi-square analyses comparing rates of utility scores below 0.95. A score of 0.95 indicates that a respondent was unwilling to trade or gamble, while a score below 0.95 indicates that a respondent was willing to trade or gamble. Utilities for caregivers and non-caregivers were compared using t-tests. After completing the initial t-tests, the utility comparisons were re-run as analyses of covariance (ANCOVA models) controlling for demographic variables that were significantly different between the two subgroups (i.e., gender and marital status in Studies 1 and 2; ethnicity and marital status in Study 3).

Sensitivity analyses were conducted in order to ascertain whether results for parents were similar to results for caregivers in general. All analyses described above were re-run twice, with two different ways of categorizing the sample. First, analyses were conducted to examine only the caregivers who were parents of dependent children, rather than the larger group of caregivers of adults and/or children that was the focus of the main analysis described above. These parents of dependent children were compared to all other respondents. Second, analyses were re-run focusing on all parents in the sample, regardless of whether their children still depended on them for care. These parents were compared to all non-parents in the sample.

\section{Results}

\section{Sample description}

In total, the current analysis was conducted with data from 364 general population respondents across the three studies, including 106 (29.1\%) categorized as caregivers and 258 (70.9\%) categorized as non-caregivers. Of the 30 participants with dependents in Study 1, 25 reported that they were parents of dependent children, while the other five reported being caregivers of adults. In Study 1, there were no statistically significant differences between caregivers $(n=30)$ and non-caregivers $(\mathrm{n}=50)$ in age, ethnicity, employment status, or education level (Table 2). However, there were significant between-group differences in gender and marital status. Compared to non-caregivers, the caregiver group was 
Table 2 Demographic characteristics

\begin{tabular}{|c|c|c|c|c|c|c|c|c|c|}
\hline \multirow[t]{2}{*}{ Characteristics } & \multicolumn{3}{|c|}{ Study 1} & \multicolumn{3}{|c|}{ Study 2} & \multicolumn{3}{|c|}{ Study 3} \\
\hline & $\begin{array}{c}\text { Caregivers } \\
(\mathrm{N}=30)\end{array}$ & $\begin{array}{c}\text { Non-caregivers } \\
(N=50)\end{array}$ & p-value ${ }^{\ddagger}$ & $\begin{array}{c}\text { Caregivers } \\
(\mathrm{N}=47)\end{array}$ & $\begin{array}{c}\text { Non-caregivers } \\
(\mathrm{N}=111)\end{array}$ & p-value ${ }^{\ddagger}$ & $\begin{array}{l}\text { Caregivers } \\
(\mathrm{N}=29)\end{array}$ & $\begin{array}{c}\text { Non-caregivers } \\
(\mathrm{N}=97)\end{array}$ & p-value ${ }^{\ddagger}$ \\
\hline Age (mean, SD) & $48.3(11.5)$ & $46.7(15.9)$ & 0.64 & $44.9(11.1)$ & $47.9(15.6)$ & 0.23 & $48.9(11.2)$ & $49.7(16.9)$ & 0.82 \\
\hline \multicolumn{10}{|l|}{ Gender (n,\%) } \\
\hline Male & $20(66.7 \%)$ & $21(42.0 \%)$ & 0.03 & 15 (31.9\%) & 65 (58.6\%) & $<0.01$ & $12(41.4 \%)$ & 48 (49.5\%) & 0.44 \\
\hline Female & $10(33.3 \%)$ & 29 (58.0\%) & & $32(68.1 \%)$ & $46(41.4 \%)$ & & $17(58.6 \%)$ & $49(50.5 \%)$ & \\
\hline \multicolumn{10}{|l|}{ Ethnicity $(n, \%)^{*}$} \\
\hline White & $15(50.0 \%)$ & 35 (70.0\%) & 0.07 & $41(87.2 \%)$ & 91 (82.0\%) & 0.42 & $10(34.5 \%)$ & $58(59.8 \%)$ & 0.02 \\
\hline Other & $15(50.0 \%)$ & 15 (30.0\%) & & $6(12.8 \%)$ & $20(18.0 \%)$ & & 19 (65.5\%) & $39(40.2 \%)$ & \\
\hline \multicolumn{10}{|l|}{ Marital status $(n, \%)$} \\
\hline Single & $8(26.7 \%)$ & 29 (58.0\%) & 0.02 & $10(21.3 \%)$ & $54(48.6 \%)$ & $<0.01$ & $9(31.0 \%)$ & $59(60.8 \%)$ & 0.02 \\
\hline $\begin{array}{l}\text { Married/Living with } \\
\text { partner }\end{array}$ & $11(36.7 \%)$ & $12(24.0 \%)$ & & $26(55.3 \%)$ & 35 (31.5\%) & & $8(27.6 \%)$ & $13(13.4 \%)$ & \\
\hline $\begin{array}{l}\text { Divorced/Separated/ } \\
\text { Widowed }\end{array}$ & $11(36.7 \%)$ & $9(18.0 \%)$ & & $11(23.4 \%)$ & $22(19.8 \%)$ & & $12(41.4 \%)$ & $25(25.8 \%)$ & \\
\hline \multicolumn{10}{|l|}{$\begin{array}{l}\text { Employment status } \\
(n, \%)^{\dagger}\end{array}$} \\
\hline Full-time work & $8(26.7 \%)$ & $12(24.0 \%)$ & 0.12 & 17 (36.2\%) & 35 (31.5\%) & 0.11 & 7 (24.1\%) & $16(16.5 \%)$ & 0.15 \\
\hline Part-time work & $10(33.3 \%)$ & 18 (36.0\%) & & $16(34.0 \%)$ & $24(21.6 \%)$ & & $12(41.4 \%)$ & $24(24.7 \%)$ & \\
\hline Unemployed & $1(3.3 \%)$ & $10(20.0 \%)$ & & $2(4.3 \%)$ & $17(15.3 \%)$ & & $3(10.3 \%)$ & 17 (17.5\%) & \\
\hline Other & $11(36.7 \%)$ & 10 (20.0\%) & & $12(25.5 \%)$ & 35 (31.5\%) & & $7(24.1 \%)$ & $40(41.2 \%)$ & \\
\hline \multicolumn{10}{|l|}{$\begin{array}{l}\text { Education Level } \\
(n, \%)\end{array}$} \\
\hline No university degree & $16(53.3 \%)$ & $25(50.0 \%)$ & 0.77 & $34(72.3 \%)$ & $64(57.7 \%)$ & 0.08 & $14(48.3 \%)$ & $44(45.4 \%)$ & 0.78 \\
\hline $\begin{array}{l}\text { University or } \\
\text { postgraduate degree }\end{array}$ & $14(46.7 \%)$ & $25(50.0 \%)$ & & $13(27.7 \%)$ & $47(42.3 \%)$ & & $15(51.7 \%)$ & $53(54.6 \%)$ & \\
\hline
\end{tabular}

*For Study 1, other ethnicity includes 13 Black, 8 Asian, and 9 other races. For Study 2, other ethnicity includes 7 Black, 8 Asian, 1 Chinese and 10 mixed races. For Study 3, other ethnicity includes 24 Black, 10 Hispanic/Latino, 10 Asian, 5 Hawaiian/Pacific Islander and 9 other races.

${ }^{\dagger}$ For Study 1, other employment includes 3 homemakers, 2 students, 15 retired and 1 disabled. For Study 2, other employment includes 8 homemakers, 9 students, 27 retired, and 3 disabled. For Study 3, other employment includes 3 homemakers, 8 students, 15 retired, 19 disabled and 1 other.

${ }^{\ddagger} \mathrm{P}$-values are for the comparison between caregivers and non-caregivers. Continuous variables were compared with $\mathrm{t}$-tests, and categorical variables were compared with chi-square analysis.

more likely to be male $(\mathrm{p}=0.03)$ and less likely to be single $(\mathrm{p}=0.02)$.

In Study 2, 47 of the 158 participants were categorized as caregivers. Of the 47,39 reported that they were parents of dependent children, four were caregivers of adults, and another four reported that they were both parents of dependent children and caregivers of adults (i.e., a total of 43 respondents were parents of dependent children). Similar to Study 1, there were no statistically significant between-group differences in age, ethnicity, employment status, or education level (Table 2). However, there were significant differences in gender and marital status. Compared to the group without dependents, the group with dependents was more likely to be female $(p<0.01)$ and married or living with a partner $(\mathrm{p}<0.01)$.

In Study 3, 29 of 126 participants were categorized as caregivers, including 19 who reported that they were parents of dependent children. Six reported being caregivers of adults, and four reported being both parents of dependent children and caregivers of adults (i.e., a total of 23 respondents were parents of dependent children). There were no statistically significant between-group differences in age, gender, employment status, or education level (Table 2). There were significant differences between caregivers and non-caregivers in ethnicity and marital status. Compared to non-caregivers, the caregiver group was more likely to be non-white $(\mathrm{p}=0.02)$ and separated $(\mathrm{p}=0.02)$.

Gender frequencies within each caregiver status group followed a different pattern in Study 1 than in Studies 2 and 3 (Table 2). Caregivers in Studies 2 and 3 were more likely to be female, whereas caregivers in Study 1 were more likely to be male. Because the gender frequencies in Study 1 were contrary to results from Studies 2 and 3, which had larger samples, this finding was confirmed by re-checking and re-tabulating gender and caregiver status from the original demographic questionnaires. This 
verification process confirmed that these gender statistics are reported correctly.

\section{Willingness to trade time or gamble}

If the respondent is unwilling to trade any time in the TTO task or unwilling to gamble in the SG task, the resulting utility will be at the ceiling, which was 0.95 in the current analysis. Compared to non-caregivers, caregivers had fewer health state utilities less than 0.95 and more responses at the ceiling, indicating less willingness to trade time or gamble. This pattern was consistent across all three studies and across almost all health states. For all health states in Studies 1 and 2 (Tables 3 and 4), a smaller percentage of caregivers had utilities less than 0.95 compared with non-caregivers. In Study 3 (Table 4), a smaller percentage of caregivers had scores less than 0.95 for 14 of 16 health states (the exceptions were health states $\mathrm{F}$ and $\mathrm{H}$ ). For example, in Study 1, while only $23.3 \%$ of caregivers were willing to trade time to avoid health state A, 46.0\% of non-caregivers were willing to trade time. Chi-square analyses found that these differences between groups were statistically significant for two health states in Study 1 and two health states in Study 3. In sum, these findings indicate a greater ceiling effect for caregivers than for noncaregivers across all three samples.

\section{Utilities}

For every health state in all three samples, caregivers had higher utilities than non-caregivers. The difference in utility scores between caregivers and non-caregivers

Table 3 Frequency and percentage of respondents with values less than $0.95^{1}$ (Study 1)

\begin{tabular}{lccc}
\hline Health states & $\begin{array}{c}\text { Caregivers } \\
(\mathbf{N}=\mathbf{3 0})\end{array}$ & $\begin{array}{c}\text { Non-caregivers } \\
\mathbf{( N = 5 0 )}\end{array}$ & p-value ${ }^{2}$ \\
\hline Time trade-off & & & \\
HS A: Mild Osteoarthritis & $7(23.3 \%)$ & $23(46.0 \%)$ & 0.04 \\
HS B: Moderate Osteoarthritis & $12(40.0 \%)$ & $31(62.0 \%)$ & 0.06 \\
HS C: Severe Osteoarthritis & $21(70.0 \%)$ & $46(92.0 \%)$ & $<0.01$ \\
HS D: Moderate Osteoarthritis & $14(46.7 \%)$ & $34(68.0 \%)$ & 0.06 \\
+ AE & & & \\
Standard gamble & & & \\
HS A: Mild Osteoarthritis & $5(17.2 \%)$ & $9(18.4 \%)$ & 0.90 \\
HS B: Moderate Osteoarthritis & $9(31.0 \%)$ & $16(32.7 \%)$ & 0.88 \\
HS C: Severe Osteoarthritis & $20(69.0 \%)$ & $40(81.6 \%)$ & 0.20 \\
HS D: Moderate Osteoarthritis & $11(37.9 \%)$ & $20(40.8 \%)$ & 0.80 \\
+ AE & & &
\end{tabular}

${ }^{1}$ Values less than 0.95 indicate willingness to trade in time trade-off choices and willingness to gamble in standard gamble choices. For the descriptive analyses in this table, ratings worse than dead were categorized as less than 0.95 .

${ }^{2}$ Based on chi-square analyses comparing caregivers and non-caregivers. $\mathrm{HS}=$ Health state.

$\mathrm{AE}=$ Adverse event ranged from 0.07 to 0.16 for TTO utilities and 0.03 to 0.17 for SG utilities in Study 1 (Table 5 ). The betweengroup difference in TTO utilities ranged from 0.06 to 0.10 in Study 2, and from 0.11 to 0.22 in Study 3 (Table 6). In seven of the eight pairwise comparisons for Study 1, as well as all comparisons for Studies 2 and 3, the mean utility difference between caregivers and non-caregivers was sufficiently large to be considered clinically important (i.e., utility difference $\geq 0.05$ [23]). The differences between caregivers and non-caregivers were statistically significant $(\mathrm{p}<0.05)$ for some health states, including health states A and $C$ in the TTO task in Study 1 and health state $C$ in the SG task in Study 1 (Table 5). In Study 2, the betweengroup difference in TTO utilities was statistically significant for all three health states (Table 6). In Study 3, the between-group difference was statistically significant for health states J and P (Table 6).

Analyses were re-run as ANCOVA models, controlling for demographic variables that were statistically significant between groups (i.e., gender and marital status in Studies 1 and 2; ethnicity and marital status in Study 3). All differences between caregivers and non-caregivers that were statistically significant in the t-tests (Tables 5 and 6) were also statistically significant in these ANCOVA models. This suggests that between-group differences were due to caregiver status rather than other demographic differences. The ANCOVA models found one additional statistically significant difference between caregivers and non-caregivers (health state B in Study 3).

\section{Sensitivity analysis: parents}

All analyses were re-run focusing on the 83 parents of dependent children ( $\mathrm{n}=25$ in Study 1; $\mathrm{n}=39$ in Study 2; $\mathrm{n}=19$ in Study 3). These analyses followed the same patterns as those for the larger caregiver group. Compared with respondents who were not parents of dependent children, these parents were less likely to trade any time in TTO tasks for every health state rated across the three studies. This difference was statistically significant for five of the 23 health states $(\mathrm{p}<0.05)$. In addition, these parents had lower TTO utility scores for every health state, with utility differences ranging from 0.06 to 0.14 in Study 1, 0.07 to 0.11 in Study 2, and 0.07 to 0.32 in Study 3. These utilities or differences were statistically significant for five of the 23 health states $(\mathrm{p}<0.05)$. The SG results in Study 1 followed similar patterns, with lower utility scores and less willingness to gamble among these parents of dependent children than among the comparison group.

These analyses were also re-run focusing on the 173 participants who reported being parents, regardless of whether their children still depended on them for care ( $\mathrm{n}=33$ in Study 1; $\mathrm{n}=89$ in Study 2; $\mathrm{n}=51$ in Study 3). These analyses followed the same patterns as those for the caregiver group and the group consisting of parents 
Table 4 Frequency and percentage of respondents with TTO utility values less than $0.95^{1}$ (Studies 2 and 3 )

\begin{tabular}{|c|c|c|c|}
\hline Health states & Caregivers & Non-caregivers & p-value ${ }^{2}$ \\
\hline \multicolumn{4}{|l|}{ Study 2 (Caregivers, $n=47$, Non-caregivers, $n=111$ ) } \\
\hline A. Adult ADHD, receiving treatment, responder & $24(51.1 \%)$ & $65(58.6 \%)$ & 0.39 \\
\hline B. Adult ADHD, receiving treatment, non-responder & $33(70.2 \%)$ & $86(77.5 \%)$ & 0.33 \\
\hline C. Adult ADHD, untreated & $34(72.3 \%)$ & $88(79.3 \%)$ & 0.34 \\
\hline \multicolumn{4}{|l|}{ Study 3 (Caregivers, $n=29$, Non-caregivers, $n=97$ ) } \\
\hline A. Basic HS (stable schizophrenia, no adverse events) & $20(69.0 \%)$ & $73(75.3 \%)$ & 0.50 \\
\hline B. Basic HS + metabolic syndrome & $22(75.9 \%)$ & $86(88.7 \%)$ & 0.08 \\
\hline C. Basic HS + weight gain & $23(79.3 \%)$ & $78(80.4 \%)$ & 0.90 \\
\hline D. Basic HS + diabetes & $21(72.4 \%)$ & $83(85.6 \%)$ & 0.10 \\
\hline E. Basic HS + hyperlipidemia & $21(72.4 \%)$ & $83(85.6 \%)$ & 0.10 \\
\hline F. Basic HS + male sexual dysfunction & $10(83.3 \%)$ & $39(81.3 \%)$ & 0.87 \\
\hline G. Basic HS + female sexual dysfunction & $13(76.5 \%)$ & $41(83.7 \%)$ & 0.51 \\
\hline H. Basic HS + male increased prolactin levels & $10(83.3 \%)$ & $40(83.3 \%)$ & 1.00 \\
\hline I. Basic HS + female increased prolactin levels & $12(70.6 \%)$ & $43(87.8 \%)$ & 0.10 \\
\hline J. Basic HS + akathisia & $22(75.9 \%)$ & $84(86.6 \%)$ & 0.17 \\
\hline K. Basic HS + tardive dyskinesia & $24(82.8 \%)$ & $90(92.8 \%)$ & 0.11 \\
\hline L. Basic HS + other extrapyramidal symptoms & $23(79.3 \%)$ & $91(93.8 \%)$ & 0.02 \\
\hline M. Basic HS + insomnia & $20(69.0 \%)$ & $83(85.6 \%)$ & 0.04 \\
\hline N. Basic HS + somnolence & $21(72.4 \%)$ & $83(85.6 \%)$ & 0.10 \\
\hline O. Basic HS + nausea & $20(69.0 \%)$ & $80(82.5 \%)$ & 0.11 \\
\hline P. Basic HS + vomiting & $21(72.4 \%)$ & $81(83.5 \%)$ & 0.18 \\
\hline
\end{tabular}

${ }^{1}$ Values less than 0.95 indicate willingness to trade in time trade-off choices.

${ }^{2}$ Based on chi-square analyses comparing caregivers and non-caregivers.

$\mathrm{HS}=$ Health state.

$\mathrm{ADHD}=$ Attention deficit/hyperactivity disorder.

TTO = Time trade-off.

Table 5 Health state utilities* (Study 1)

\begin{tabular}{|c|c|c|c|c|c|c|c|c|c|}
\hline \multirow[t]{2}{*}{ Health states } & \multicolumn{3}{|c|}{ Caregivers $(\mathrm{N}=30)$} & \multicolumn{3}{|c|}{ Non-caregivers $(\mathrm{N}=50)$} & \multirow[t]{2}{*}{ Difference } & \multirow[t]{2}{*}{ t statistic } & \multirow[t]{2}{*}{ p-value } \\
\hline & $\mathrm{n}^{\dagger}$ & Mean (SD) & Min-Max & $\mathrm{n}^{\dagger}$ & Mean (SD) & Min-Max & & & \\
\hline \multicolumn{10}{|l|}{ Time trade-off } \\
\hline HS A: Mild Osteoarthritis & 30 & $0.92(0.06)$ & $0.75-0.95$ & 50 & $0.85(0.13)$ & $0.45-0.95$ & $0.07(0.11)$ & 3.2 & $<0.01$ \\
\hline HS B: Moderate Osteoarthritis & 30 & $0.84(0.19)$ & $0.05-0.95$ & 49 & $0.76(0.23)$ & $0.00-0.95$ & $0.08(0.22)$ & 1.6 & 0.11 \\
\hline HS C: Severe Osteoarthritis & 24 & $0.69(0.28)$ & $0.15-0.95$ & 42 & $0.53(0.29)$ & $0.05-0.95$ & $0.16(0.28)$ & 2.3 & 0.03 \\
\hline HS D: Moderate Osteoarthritis + AE & 30 & $0.82(0.21)$ & $0.05-0.95$ & 48 & $0.72(0.26)$ & $0.00-0.95$ & $0.10(0.25)$ & 1.8 & 0.07 \\
\hline \multicolumn{10}{|l|}{ Standard gamble } \\
\hline HS A: Mild Osteoarthritis & 29 & $0.93(0.05)$ & $0.75-0.95$ & 49 & $0.90(0.12)$ & $0.45-0.95$ & $0.03(0.10)$ & 1.4 & 0.16 \\
\hline HS B: Moderate Osteoarthritis & 29 & $0.89(0.10)$ & $0.60-0.95$ & 48 & $0.84(0.20)$ & $0.25-0.95$ & $0.05(0.17)$ & 1.5 & 0.13 \\
\hline HS C: Severe Osteoarthritis & 24 & $0.79(0.19)$ & $0.35-0.95$ & 40 & $0.61(0.33)$ & $0.05-0.95$ & $0.17(0.28)$ & 2.7 & $<0.01$ \\
\hline HS D: Moderate Osteoarthritis + AE & 29 & $0.88(0.11)$ & $0.60-0.95$ & 47 & $0.81(0.23)$ & $0.15-0.95$ & $0.07(0.20)$ & 1.7 & 0.09 \\
\hline
\end{tabular}

"Based on t-tests comparing means of the two groups. Utility ratings worse than dead are not included in this table. In Study 1 , a precise utility value was not obtained when a respondent considered a health state to be worse than dead (i.e., utility $<0$ ). Therefore, participants who considered a health state to be worse than dead have missing data for that health state in this table.

${ }^{\dagger}$ In Study 1, sample size varied for each individual health state because a specific utility value was not derived if a respondent believed that health state was worse than being dead.

$\mathrm{HS}=$ Health state.

$\mathrm{AE}=$ Adverse event 
Table 6 Time trade-off utilities* (Studies 2 and 3)

\begin{tabular}{|c|c|c|c|c|c|c|c|c|c|}
\hline \multirow[t]{2}{*}{ Health states } & \multicolumn{3}{|c|}{ Caregivers $(\mathrm{N}=47)$} & \multicolumn{3}{|c|}{ Non-caregivers $(\mathrm{N}=111)$} & \multirow[t]{2}{*}{ Difference } & \multirow[t]{2}{*}{ t statistic } & \multirow[t]{2}{*}{ p-value } \\
\hline & n & Mean (SD) & Min-Max & n & Mean (SD) & Min-Max & & & \\
\hline \multicolumn{10}{|l|}{ Study 2} \\
\hline A. Adult ADHD, receiving treatment, responder & 47 & $0.87(0.11)$ & $0.45-0.95$ & 111 & $0.80(0.19)$ & $-0.05-0.95$ & $0.06(0.17)$ & 2.7 & $<0.01$ \\
\hline B. Adult ADHD, receiving treatment, non-responder & 47 & $0.75(0.21)$ & $0.05-0.95$ & 111 & $0.65(0.30)$ & $-0.75-0.95$ & $0.10(0.28)$ & 2.5 & 0.02 \\
\hline C. Adult ADHD, untreated & 47 & $0.73(0.23)$ & $0.05-0.95$ & 111 & $0.64(0.30)$ & $-0.65-0.95$ & $0.10(0.28)$ & 2.2 & 0.03 \\
\hline \multicolumn{10}{|l|}{ Study 3} \\
\hline A. Basic HS (stable schizophrenia, no adverse events) & 29 & $0.68(0.35)$ & $-0.55-0.95$ & 97 & $0.56(0.45)$ & $-0.75-0.95$ & $0.11(0.43)$ & 1.3 & 0.21 \\
\hline B. Basic HS + metabolic syndrome & 29 & $0.62(0.35)$ & $-0.55-0.95$ & 97 & $0.44(0.49)$ & $-0.85-0.95$ & $0.18(0.46)$ & 1.9 & 0.06 \\
\hline C. Basic HS + weight gain & 29 & $0.64(0.36)$ & $-0.55-0.95$ & 97 & $0.51(0.47)$ & $-0.85-0.95$ & $0.12(0.45)$ & 1.3 & 0.19 \\
\hline D. Basic HS + diabetes & 29 & $0.58(0.39)$ & $-0.55-0.95$ & 97 & $0.43(0.50)$ & $-0.95-0.95$ & $0.15(0.48)$ & 1.5 & 0.13 \\
\hline E. Basic HS + hyperlipidemia & 29 & $0.61(0.38)$ & $-0.55-0.95$ & 97 & $0.47(0.47)$ & $-0.85-0.95$ & $0.13(0.45)$ & 1.4 & 0.16 \\
\hline F. Basic HS + male sexual dysfunction ${ }^{\dagger}$ & 12 & $0.57(0.29)$ & $0.05-0.95$ & 48 & $0.47(0.45)$ & $-0.85-0.95$ & $0.11(0.43)$ & 0.8 & 0.44 \\
\hline G. Basic HS + female sexual dysfunction ${ }^{\dagger}$ & 17 & $0.61(0.43)$ & $-0.55-0.95$ & 49 & $0.45(0.52)$ & $-0.75-0.95$ & $0.16(0.50)$ & 1.2 & 0.25 \\
\hline H. Basic HS + male increased prolactin levels ${ }^{\dagger}$ & 12 & $0.62(0.27)$ & $0.05-0.95$ & 48 & $0.44(0.47)$ & $-0.85-0.95$ & $0.17(0.44)$ & 1.7 & 0.10 \\
\hline I. Basic HS + female increased prolactin levels ${ }^{\dagger}$ & 17 & $0.63(0.41)$ & $-0.55-0.95$ & 49 & $0.44(0.50)$ & $-0.75-0.95$ & $0.19(0.48)$ & 1.4 & 0.17 \\
\hline J. Basic HS + akathisia & 29 & $0.62(0.35)$ & $-0.55-0.95$ & 97 & $0.43(0.51)$ & $-0.85-0.95$ & $0.19(0.48)$ & 2.3 & 0.03 \\
\hline K. Basic HS + tardive dyskinesia & 29 & $0.47(0.49)$ & $-0.85-0.95$ & 97 & $0.25(0.60)$ & $-0.95-0.95$ & $0.22(0.58)$ & 1.8 & 0.07 \\
\hline L. Basic HS + other extrapyramidal symptoms & 29 & $0.41(0.52)$ & $-0.85-0.95$ & 97 & $0.25(0.57)$ & $-0.95-0.95$ & $0.16(0.56)$ & 1.4 & 0.17 \\
\hline M. Basic HS + insomnia & 29 & $0.65(0.38)$ & $-0.55-0.95$ & 97 & $0.49(0.47)$ & $-0.85-0.95$ & $0.16(0.45)$ & 1.7 & 0.09 \\
\hline N. Basic HS + somnolence & 29 & $0.64(0.36)$ & $-0.55-0.95$ & 97 & $0.48(0.47)$ & $-0.85-0.95$ & $0.16(0.44)$ & 1.7 & 0.10 \\
\hline O. Basic HS + nausea & 29 & $0.62(0.44)$ & $-0.65-0.95$ & 97 & $0.50(0.48)$ & $-0.95-0.95$ & $0.11(0.47)$ & 1.1 & 0.26 \\
\hline P. Basic HS + vomiting & 29 & $0.65(0.36)$ & $-0.55-0.95$ & 97 & $0.45(0.51)$ & $-0.95-0.95$ & $0.20(0.48)$ & 2.4 & 0.02 \\
\hline
\end{tabular}

"Based on t-tests comparing means of the two groups.

${ }^{\dagger}$ In Study 3, sample size was smaller for health states representing sexual dysfunction and increased prolactin because each participant rated only the gender-specific health state.

$\mathrm{HS}=$ Health state.

$A D H D=$ Attention deficit/hyperactivity disorder.

of dependent children. Compared with non-parents, these parents were less likely to trade any time in TTO tasks for every health state rated across the three studies. This difference was statistically significant for four of the 23 health states $(\mathrm{p}<0.05)$. In addition, these parents had lower TTO utility scores than non-parents for every health state, with utility differences ranging from 0.05 to 0.19 in Study 1, 0.03 to 0.09 in Study 2, and 0.03 to 0.09 in Study 3. These utilities or differences were statistically significant for seven of the 23 health states $(p<0.05)$. The SG results in Study 1 followed similar patterns, with lower utility scores and less willingness to gamble among parents than among non-parents.

\section{Discussion}

Taken together, results across three independent data sets for a wide range of health states provide strong support for the hypothesis that being a caregiver influences the way one responds in a TTO health state valuation task. Compared to respondents who were not caregivers, caregivers tended to be less willing to trade any time in the TTO tasks. Furthermore, when caregivers did trade, they tended to trade less time, resulting in consistently higher utility scores than non-caregivers. The difference between caregivers and non-caregivers in health state utility was often quite large, with between-group differences up to 0.22 on the standard utility scale anchored to 0 (dead) and 1 (full health). Utility differences of this magnitude are large enough to have a substantial impact on the outcome of a cost-utility model. Thus, caregiver status may be an important demographic variable to consider when collecting, interpreting, or using utility data. The current results also serve as a reminder that health state utilities are shaped not only by the health states themselves, but also by characteristics of the respondent valuing the health states.

SG data were not available in the data sets for Studies 2 or 3, but in Study 1, caregiver status appeared to have less influence on SG responses than on TTO responses. For example, caregiver status had a less pronounced impact on willingness to gamble in SG than on willingness to trade in TTO (Table 3). In addition, mean differences 
in utility scores between caregivers and non-caregivers were smaller for SG than TTO for three of the four health states in Study 1 (Table 5). Perhaps the SG task is less susceptible than TTO to the respondent's current life situation because of the abstract nature of the task. In the TTO, participants may consider the direct impact of shorter lifespan on their dependents, whereas the probabilities involved in the SG task may not lead respondents to think of their own responsibilities as directly. Still, SG utilities had a smaller utility range for caregivers than for non-caregivers, the caregivers did have statistically significantly higher SG utilities for health state C, and between-group differences for health states B and D may be considered clinically important (Table 5 [23]). In sum, while results of the TTO task appear to be more strongly influenced by caregiver status, SG responses may also be affected. Future research is needed to clarify the impact of caregiver status on SG responses.

Current findings add to results reported by van der Pol \& Shiell [15] suggesting that people are less willing to trade time in the hypothetical TTO task if they know that others are directly dependent on them. Whereas the van der Pol \& Shiell [15] study focused only on a sample of 30 parents of young children, the current study included both parents of dependent children and individuals who considered themselves to be caregivers of an adult. This broader group was used because respondents in previous utility assessment studies (conducted by authors of the current studies) have mentioned the impact of caring for an elderly or disabled relative. However, the great majority of caregivers in the current study cared for children rather than adults (i.e., 83 of 106 caregivers; $78.3 \%)$. As a sensitivity analysis, current analyses were re-run in two other subgroups: including parents of dependent children and parents of any child regardless of whether the child is still dependent. Results of these sensitivity analyses followed the same general pattern as results of the primary analysis. The parent subgroups were generally less willing to trade time (i.e., resulting in higher utility scores) than comparison groups. Future research, perhaps involving qualitative interviews asking participants to explain their TTO responses, is needed to explore the separate influence, if any, of parenting and other types of caregiving on utility scores. The current analysis may also be replicated in larger samples with various types of caregivers, including larger numbers of caregivers of adults.

Parenting and other types of caregiving may reduce willingness to trade due to increased altruism and a sense of responsibility for another individual's wellbeing. In addition, even if a child is grown, being a parent may reduce willingness to trade because parents may want to live longer to experience specific milestones in their child's life. For example, parents have reported that they wanted to live long enough to see their child's wedding or graduation when responding in previous TTO studies conducted by the authors of the current study. Such extrinsic goals, which occur at a specific point in the future, have been proposed as one factor that could limit willingness to trade time in TTO or gamble in SG [24].

Current results also have implications for the conceptual foundations of utility assessments. The standard preference elicitation and quality-adjusted life year (QALY) models ask respondents to evaluate health states based on the effects on their own welfare. However, results reported in this paper and elsewhere in the literature suggest that respondents also consider the effects of their health on the welfare of others when valuing health states. Several authors have developed conceptual frameworks that broaden the utility model to take into account not only the preferences of respondents for their own health outcomes, but also their preferences for effects on other people such as spouses, children, and adult dependents. These frameworks are often referred to as a family or household utility function [25-27]. Current findings suggest that future research to develop and implement these family or household utility approaches could be useful, although this methodology could be complex, and the scores may not be entirely comparable to conventional utilities.

Study limitations related to sample selection should be acknowledged. During screening, efforts were made to avoid over-representing respondents from any particular age, ethnic/racial background, gender, or employment group. However, recruitment was not stratified or designed to be nationally representative in any of the three studies used for this secondary analysis. Furthermore, the recruitment strategy, requiring that all study participants respond to newspaper or online advertisements, could have introduced selection bias. The extent to which current results may differ from utilities derived from a nationally representative sample in the US, UK, or any other country is not known.

Another potential limitation is that a respondent's personal experience with the condition described in health states could influence utility scores. In Studies 2 and 3, none of the participants were ever diagnosed with the condition described in the health states (ADHD and schizophrenia, respectively), although it is possible that they knew people who had received these diagnoses. It is not known whether participants in Study 1 had ever been diagnosed with the condition described in the health states (osteoarthritis). Personal knowledge of the conditions being rated could have an impact on utility scores, and it is not known whether this was a confounding factor in the current analysis.

It is also possible that aspects of the interview methodology could have introduced bias. For example, although 
health states were initially presented to participants in random order during ranking or VAS introductory tasks, health states were then presented in the TTO tasks in the order in which participants ranked them. At multiple points during the interviews and at the end of the interviews, participants were asked to review and compare all of their utility scores in order to minimize ordering effects and learning effects that could affect differences among health states. Furthermore, if there were any ordering or learning effects, any resulting biases would likely be the same for caregivers and non-caregivers, and consequently, this issue is unlikely to have an impact on the issues examined in the current secondary analysis.

\section{Conclusions}

In sum, the current study indicates that parenting and potentially other types of caregiving appear to influence TTO utilities. Subsequent research may be conducted to replicate current findings in independent cohorts of parents with dependent children as well as among caregivers of adults. In future TTO studies, it may also be useful to assess parenting and adult caregiving status along with other demographic variables such as gender, age, and education. Samples with higher percentages of parents or caregivers may tend to yield higher utility scores. Thus, it may be helpful to know these respondent characteristics when interpreting data and comparing utilities across multiple studies.

\section{Abbreviations}

TTO: Time trade-off; SG: Standard gamble; ADHD: Attention-deficit/ hyperactivity disorder; NICE: National institute for health and care excellence; VAS: Visual analogue scale; QALY: Quality-adjusted life year; HS: Health states.

\section{Competing interests}

Funding for this study was provided by Eli Lilly \& Co. Three of the authors (Kristina Boye, Joseph Johnston, and Lee Bowman) are employees of Lilly, but their input into the conceptualization and interpretation of this study represented their own opinions, rather than those of the company. Louis Matza and Jessica Jordan are employees of Evidera, a company that received funding from Lilly for this research. David Feeny received funding from Lilly for time spent contributing to this research.

\section{Authors' contributions}

LM was the primary writer of this manuscript, and he directed the study including the study design, health state development (for studies 2 and 3), data collection, data analysis, and data interpretation. KB, JAJ, LB, and DF made substantial contributions to the study design, data interpretation, and resulting manuscript. JBJ co-wrote sections of the manuscript and assisted with study design. All authors provided input on multiple drafts of the manuscript and approval of the final draft. All authors read and approved the final manuscript.

\section{Acknowledgments}

Katie Devine contributed to study design for Study 2, Julie Birt contributed to study design for Study 3, and Virginia Sutton Haynes contributed to study design for Studies 2 and 3. The authors would like to thank Christine Thompson for statistical programming; Kelly McDaniel, Evan Davies, Amanda Landrian, Katie Devine, Andrew Palsgrove, Zaneta Balantac, Anna Tate, Jacqueline Kostelec, Mary Kay Margolis, Ann Peyser, and Dylan Trundell for assistance with data collection; and Aria Gray, Emily Sargent, and Amara Tiebout for production assistance. Funding for this study was provided by Eli Lilly \& Co.

\section{Author details}

'Senior Research Scientist, Outcomes Research, Evidera, Bethesda, MD, USA.

${ }^{2}$ Sr. Research Advisor, Global Health Outcomes, Diabetes, Eli Lilly and Company, Indianapolis, IN, USA. ${ }^{3}$ Department of Economics (Professor Emeritus), University of Alberta, Edmonton, AB, Canada. ${ }^{4}$ Medical Fellow, Global Health Outcomes, Eli Lilly and Company, Indianapolis, IN, USA. ${ }^{5}$ Research Fellow, Global Health Outcomes, Oncology, Eli Lilly and Company, Indianapolis, IN, USA. ${ }^{6}$ Senior Research Associate, Outcomes Research, Evidera, Bethesda, MD, USA.

Received: 28 August 2013 Accepted: 12 March 2014

Published: 9 April 2014

\section{References}

1. Rowen D, Brazier J: Health utility measurement. In The Oxford Handbook of Health Economics. Edited by Glied S, Smith PC. New York: Oxford University Press; 2011:788-813.

2. Torrance GW: Measurement of health state utilities for economic appraisal. J Health Econ 1986, 5:1-30.

3. Torrance GW, Furlong W, Feeny D: Health utility estimation. Expert Rev Pharmacoecon Outcomes Res 2002, 2:99-108.

4. Ashby J, O'Hanlon M, Buxton MJ: The time trade-off technique: how do the valuations of breast cancer patients compare to those of other groups? Qual Life Res 1994, 3:257-265.

5. Baker R, Robinson A: Responses to standard gambles: are preferences 'well constructed'? Health Econ 2004, 13:37-48.

6. Badia X, Monserrat S, Roset M, Herdman M: Feasibility, validity and testretest reliability of scaling methods for health states: the visual analogue scale and the time trade-off. Qual Life Res 1999, 8:303-310.

7. Van Nooten FE, Koolman X, Brouwer WB: The influence of subjective life expectancy on health state valuations using a 10 year TTO. Health Econ 2009, 18:549-558.

8. Bursztajn $\mathrm{H}$, Hamm RM: The clinical utility of utility assessment. Med Decis Making 1982, 2:161-165.

9. Mrus JM, Sherman KE, Leonard AC, Sherman SN, Mandell KL, Tsevat J: Health values of patients coinfected with HIV/hepatitis C: are two viruses worse than one? Med Care 2006, 44:158-166.

10. Yi MS, Britto MT, Sherman SN, Moyer MS, Cotton S, Kotagal UR, Canfield D, Putnam FW, Carlton-Ford S, Tsevat J: Health values in adolescents with or without inflammatory bowel disease. J Pediatr 2009, 154:527-534.

11. Dolan P, Roberts J: To what extent can we explain time trade-off values from other information about respondents? Soc Sci Med 2002, 54:919-929.

12. Stiggelbout AM, Kiebert GM, Kievit J, Leer JW, Habbema JD, De Haes JC: The "utility" of the Time Trade-Off method in cancer patients: feasibility and proportional Trade-Off. J Clin Epidemiol 1995, 48:1207-1214.

13. Rosen $A B$, Tsai JS, Downs SM: Variations in risk attitude across race, gender, and education. Med Decis Making 2003, 23:511-517.

14. Tsuchiya A, Ikeda S, Ikegami N, Nishimura S, Sakai I, Fukuda T, Hamashima C, Hisashige A, Tamura M: Estimating an EQ-5D population value set: the case of Japan. Health Econ 2002, 11:341-353.

15. van der Pol M, Shiell A: Extrinsic goals and time tradeoff. Med Decis Making 2007, 27:406-413.

16. Brazier J: Valuing health states for use in cost-effectiveness analysis. Pharmacoeconomics 2008, 26:769-779.

17. NICE (National Institute for Health and Clinical Excellence): Process and methods guides. In Guide to the methods of technology appraisal 2013. London, UK: National Institute for Care Excellence (NICE); 2013. http://www. nice.org.uk/media/D45/1E/GuideToMethodsTechnologyAppraisal2013.pdf. Accessed: August 19, 2013

18. PBAC (Pharmaceutical Benefits Advisory Committee): Guidelines for preparing submissions to PBAC, Version 4.3.2.: PBAC. PBAC (Pharmaceutical Benefits Advisory (ommittee); 2008.

19. Feeny D, Blanchard CM, Mahon JL, Bourne R, Rorabeck C, Stitt L, Webster-Bogaert S: The stability of utility scores: test-retest reliability and the interpretation of utility scores in elective total hip arthroplasty. Qual Life Res 2004, 13:15-22.

20. Laupacis A, Bourne R, Rorabeck C, Feeny D, Wong C, Tugwell P, Leslie K, Bullas $R$ : The effect of elective total hip replacement on health-related quality of life. J Bone Joint Surg Am 1993, 75:1619-1626.

21. Brazier J, Ratcliffe J, Salomon JA, Tsuchiya A: Measuring and Valuing Health benefits for Economic Evaluation. New York: Oxford University Press; 2007. 
22. Dolan P: Modeling valuations for EuroQol health states. Med Care 1997, 35:1095-1108

23. Feeny D: Preference-based measures: utility and quality-adjusted life years. In Assessing Quality of Life in Clinical Trials. Edited by Fayers P, Hays R. New York: Oxford University Press; 2005:405-431.

24. Hazen GB, Schwartz A: Incorporating extrinsic goals into decision and cost-effectiveness analyses. Med Decis Making 2009, 29:580-589.

25. Basu A, Meltzer D: Implications of spillover effects within the family for medical cost-effectiveness analysis. J Health Econ 2005, 24:751-773.

26. Prosser LA, Hammitt JK, Keren R: Measuring health preferences for use in cost-utility and cost-benefit analyses of interventions in children: theoretical and methodological considerations. Pharmacoeconomics 2007, 25:713-726.

27. Ungar WJ: Challenges in health state valuation in paediatric economic evaluation: are QALYs contraindicated? Pharmacoeconomics 2011, 29:641-652.

doi:10.1186/1477-7525-12-48

Cite this article as: Matza et al:: Impact of caregiver and parenting status on time trade-off and standard gamble utility scores for health state descriptions. Health and Quality of Life Outcomes 2014 12:48.

\section{Submit your next manuscript to BioMed Central and take full advantage of:}

- Convenient online submission

- Thorough peer review

- No space constraints or color figure charges

- Immediate publication on acceptance

- Inclusion in PubMed, CAS, Scopus and Google Scholar

- Research which is freely available for redistribution 